\title{
PHARMACOLOGY
}

\section{Machine learning identifies mitophagy inducers that ameliorate pathology in animal models of Alzheimer's disease}

Xie, C., Zhuang, X-X., Niu, Z., Ai, R. et al. Nat. Biomed. Eng. 6, 76-93 (2022)

Alzheimer's disease $(\mathrm{AD})$ is characterized by the presence of extracellular plaques and intracellular neurofibrillary tangles (NFTs) in the brain, resulting from the aggregation of misfolded amyloid beta (A $\beta)$ peptides and protein Tau, respectively. Recent studies have implicated impaired mitophagy in the etiology of AD. Mitophagy is a type of autophagy, whereby damaged mitochondria are engulfed in mitophagosomes and fused to lysosomes where they get eliminated. This process is essential to prevent the accumulation of dysfunctional mitochondria and maintain the integrity of the cell. Identifying new compounds that induce mitophagy could therefore have a great therapeutic value in neurodegenerative disease.

In a new study, Xie. et al., describe the identification of new mitophagy inducers using an Artificial Intelligence (AI)-aided model and a workflow to test their potential in alleviating AD pathology and memory loss in nematode and rodent models, using molecular biology, imaging and behavioural tools. To screen for mitophagy inducers, the investigators used a combinational AI approach to overcome the lack of large numbers of reference compounds that a typical AI approach would require. They used the CheMBL and ZINC natural product databases as a pre-training dataset, and following model development, they screened a library of 3,274 natural compounds. Eighteen molecules were selected and further tested in vitro. Of the eight molecules that showed the ability to induce mitophagy in HeLa cells, two compounds, Kaempferol and Rhapontigenin, also robustly induced neuronal mitophagy in a C.elegans mitophagy reporter strain.

Next, the researchers used an aversive olfactory learning chemotaxis assay to test the effects of the mitophagy inducers on memory in nematode models of $\mathrm{AD}$, which revealed that compounds Kaem and Rhap stimulated mitophagy and improved associative memory in $\mathrm{A} \beta$ and tau C.elegans models. Memory retention was mediated by an improvement in function and survival of glutamatergic and cholinergic neurons, and was associated with a reduction in $A \beta$ production and pathological Tau aggregates. Similarly, Kaem and Rhap improved spatial learning and memory in a mouse model of $\mathrm{AD}$ and alleviated both $A \beta$ and Tau pathologies.

This study identified two mitophagy inducers that inhibit both $\mathrm{A} \beta$ and Tau-related AD pathologies in two species, which supports further testing of the compounds in a clinical setting. Additional mitophagy inducers could be discovered by applying the AI-driven screening approach and cross-species validation workflow described here to larger drug libraries.

\section{Anna Melidoni}

Published online: 1 March 2022 https://doi.org/10.1038/s41684-022-00932-1

\section{++++
ENVIGO \\ ENVIGO}

\section{Custom diets as a research tool}

\section{Teklad diets support reliable, repeatable research results}

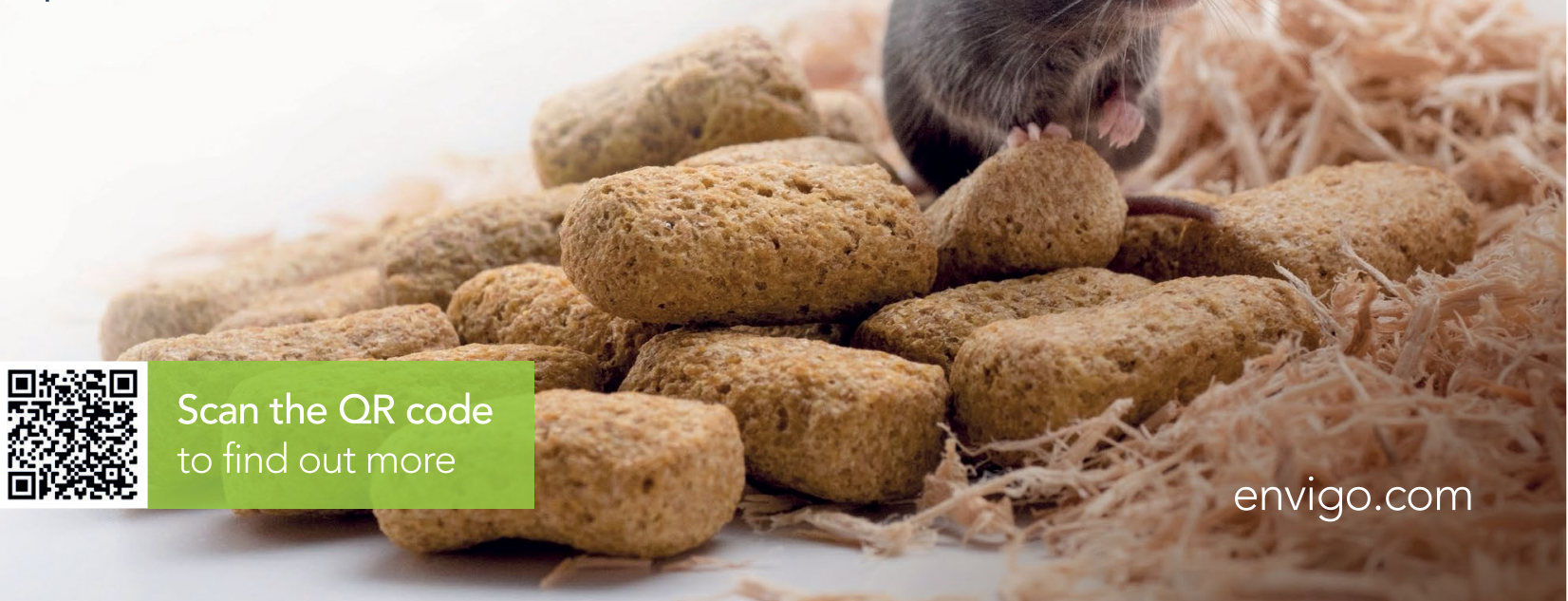

